\title{
Diagnostic electrophysiological study in a highly trained young woman with presyncopal symptoms during exercise: a case report
}

\author{
Fernando de la Guía-Galipienso ${ }^{1,2}$, Fabian Sanchis-Gomar ${ }^{3}$, Aurelio Quesada-Dorador ${ }^{4,5}$ \\ ${ }^{1}$ Cardiology Service of Marina Baixa Hospital, Alicante, Spain; ${ }^{2}$ REMA Sports Cardiology Clinic, Denia, Alicante, Spain; ${ }^{3}$ Department of Physiology, \\ Faculty of Medicine, University of Valencia and INCLIVA Biomedical Research Institute, Valencia, Spain; ${ }^{4}$ Arrhythmia Unit, Cardiology Service, \\ General University Hospital Consortium of Valencia, Valencia, Spain; ${ }^{5}$ School of Medicine, Catholic University of Valencia San Vicente Mártir, \\ Valencia, Spain \\ Correspondence to: Fabian Sanchis-Gomar, MD, PhD. Department of Physiology, Faculty of Medicine, University of Valencia, Av. Blasco Ibáñez, 15, \\ 46010 Valencia, Spain. Email: fabian.sanchis@uv.es.
}

\begin{abstract}
Right ventricular outflow tract (RVOT) ventricular tachycardia (VT) is frequent and occurs in patients without structural heart disease, especially in highly trained athletes. Most of the studies on cardiac adaptations to exercise have been investigated in male athletes. Women, however, are increasingly participating in sports and electrical and structural adaptations in male and female athletes differ significantly. These cardiac adaptations dissimilarities between males and females have potential implications in diagnosing certain types of arrhythmias. We present here a case of a 35-year-old highly-trained woman endurance athlete that attended the clinic complaining about chest pain and dyspnea on exertion, dizziness and presyncope occurring during maximum-intensity exercise training sessions. An exercise stress testing was performed on cycle ergometer. The test elapsed normally until the patient reached a heart rate of 169 bpm, when she presented identical symptoms to those described during the first interview in the clinic. A wide-complex and notched QRS tachycardia was observed in the inferior leads, inferior axis leads and transition from leads V4 to V5, suspending the test immediately. The patient was referred to perform an electrophysiological study and eventually radiofrequency catheter ablation in order to eliminate the culprit VT. Precocity occurred in the posterior lateral wall of the RVOT, immediately below the pulmonary valve. Radiofrequency application in the arrhythmogenic focus suppressed all ectopic activity despite maintaining isoproterenol infusion. After 30 minutes, the effect was maintained, and the ectopic focus was successfully ablated. The recognition of this clinical entity in females may be challenging since cardiac remodeling in response to exercise may be invaluable due to their biological, anatomical, and hormonal characteristics. In effect, electrical and structural adaptations in males and females may differ considerably. Both exercise stress testing and diagnostic electrophysiological study represent essential and invaluable tools to reach a final diagnosis, especially in highly trained females.
\end{abstract}

Keywords: Exercise; woman; cardiac remodeling; ventricular tachycardia; catheter ablation; case report

Submitted Apr 23, 2020. Accepted for publication Nov 27, 2020.

doi: 10.21037/atm-20-3492

View this article at: http://dx.doi.org/10.21037/atm-20-3492

\section{Introduction}

Right ventricular outflow tract (RVOT) ventricular tachycardia (VT) is the most frequent among the outflow tract tachycardias and occurs in patients without structural heart disease, particularly in highly trained athletes (1).
Nonetheless, most of the studies on cardiac adaptations to exercise have been investigated in male athletes and International recommendations for the interpretation of the athlete's electrocardiogram (ECG) are mostly based on those studies (2). Recent studies show that sex has a significant effect on ECG patterns in athletes and 
available data also suggest that female endurance athletes show smaller absolute cardiac dimensions than male (3), except right ventricular (RV) cavity size indexed for body surface area (BSA), which is higher in female than male athletes (4). Males and females have important biological, anatomical, and hormonal differences, including hence cardiac adaptations in response to exercise. Accordingly, electrical and structural adaptations in male and female athletes differ significantly (5). Women are increasingly participating in sports and a better understanding of cardiac physiopathological adaptations to exercise in female athletes is needed (5) in order to identify and treat specific high-intensity long-term exercise-related abnormalities successfully. This case exemplifies the cardiac adaptations dissimilarities between males and females in a highly trained woman and its potential implications on the patient's quality of life and diagnosis. We present the following article in accordance with the CARE reporting checklist (available at http://dx.doi.org/10.21037/atm-20-3492).

\section{Case presentation}

A 35-year-old high-level endurance female athlete (weight $59 \mathrm{~kg}$, height $168 \mathrm{~cm}$; BMI $20.9 \mathrm{~kg} / \mathrm{m} 2$; resting blood pressure $105 / 57 \mathrm{mmHg}$; resting heart rate $66 \mathrm{bpm}$ ) attended the clinic complaining about chest pain and dyspnea on exertion, dizziness and presyncope occurring during maximum-intensity exercise training sessions. She had been performing 15 hours/week of exercise endurance training for 20 years until symptoms' presentation. No history of cardiovascular disease, cardiovascular risk factors and no medical-surgical history of interest were reported. She denied the use of doping and/or stimulants, particularly caffeine. She had never smoked and other risk factors such as diabetes and hypertension were ruled out. No family history of sudden death, syncope, or known cardiac disease was reported.

Symptoms began about 2 years earlier and had been progressively worsening palpitations, lasting for 3 hours. The symptoms force her to stop physical exercise training immediately, improving these symptoms after 5-10 minutes of rest and resuming exercise training, but always at lower intensities. Due to this symptomatology, the patient had been previously visited three other specialists with the result of 3 different consecutive diagnoses: (I) musculoskeletal pain or costochondritis, (II) anxiety, (III) probable bronchial asthma. Ibuprofen $400 \mathrm{mg}$ every 12 hours was prescribed to treat the first diagnosis, while bromazepam $1.5 \mathrm{mg}$ daily was prescribed to treat the second one. No treatment was initially prescribed to treat the last suggested diagnosis.

The physical exam and the 12-lead baseline ECG did not report any abnormality; no premature ventricular contractions (PVCs), left bundle branch block (LBBB) pattern or positivity in inferior leads were recorded (Figure 1). Moreover, other workup studies, including blood tests (thyroid function, electrolyte and endocrine tests) and transthoracic echocardiography (TTE), were unremarkable. In brief, TTE showed normal left ventricular (LV) (LV endsystolic diameter $40.5 \mathrm{~mm})$ and wall thickness $(8 \mathrm{~mm})$ as well as normal LV systolic [left ventricular ejection fraction (LVEF) 73\%] and diastolic functions. Color flow Doppler and RV parameters [tricuspid annular plane systolic excursion $($ TAPSE) $=25 \mathrm{~mm}$; RV end-systolic diameter $=29.5 \mathrm{~mm}$ ] were within normal values.

Thereafter, an exercise stress testing was performed on cycle ergometer. The test elapsed normally until the patient reached a heart rate of $169 \mathrm{bpm}$, power in watts of 185 (3.1 watts $/ \mathrm{kg}$ ), 11.6 metabolic equivalents (METs), and $\mathrm{VO}_{2 \max }$ of $2.43 \mathrm{~L} / \mathrm{min}$ when she presented identical symptoms to those described during the first interview in the clinic. A wide-complex and notched QRS tachycardia was observed in the inferior leads, inferior axis leads and transition from leads V4 to V5 (Figure 2), suspending the test immediately. Symptoms disappeared after 50 seconds and regular sinus rhythm was concurrently restored. This finding prompted us to instruct the athlete to cease exercise training and recommended further evaluation, including cardiac magnetic resonance imaging (cMRI) and 72-hour Holter monitoring. The patient was free of symptoms whilst exercise training was interrupted and no medication was prescribed during this period. Late gadolinium enhancement (LGE)-cMRI showed normal cardiac morphology, with normal LV and RV function, and no evidence of active inflammation, fibrosis or RVOT scars, which also ruled out the presence of structural heart disease. No PVCs or other pathological electrocardiographic patterns were recorded in the 72-hour Holter monitoring.

Although PVCs, LBBB, positivity in inferior leads, fibrosis or RVOT scars were not detected, given the results obtained in the exercise stress test and the suspicion of RVOT-VT, the patient was referred to another treatment center to perform an electrophysiological study and eventually radiofrequency catheter ablation in order to eliminate the culprit VT. Standardized programmed and continuous atrial and ventricular stimulation did not induce sustained arrhythmias, only occasional extrasystoles. Isoproterenol infusion and 


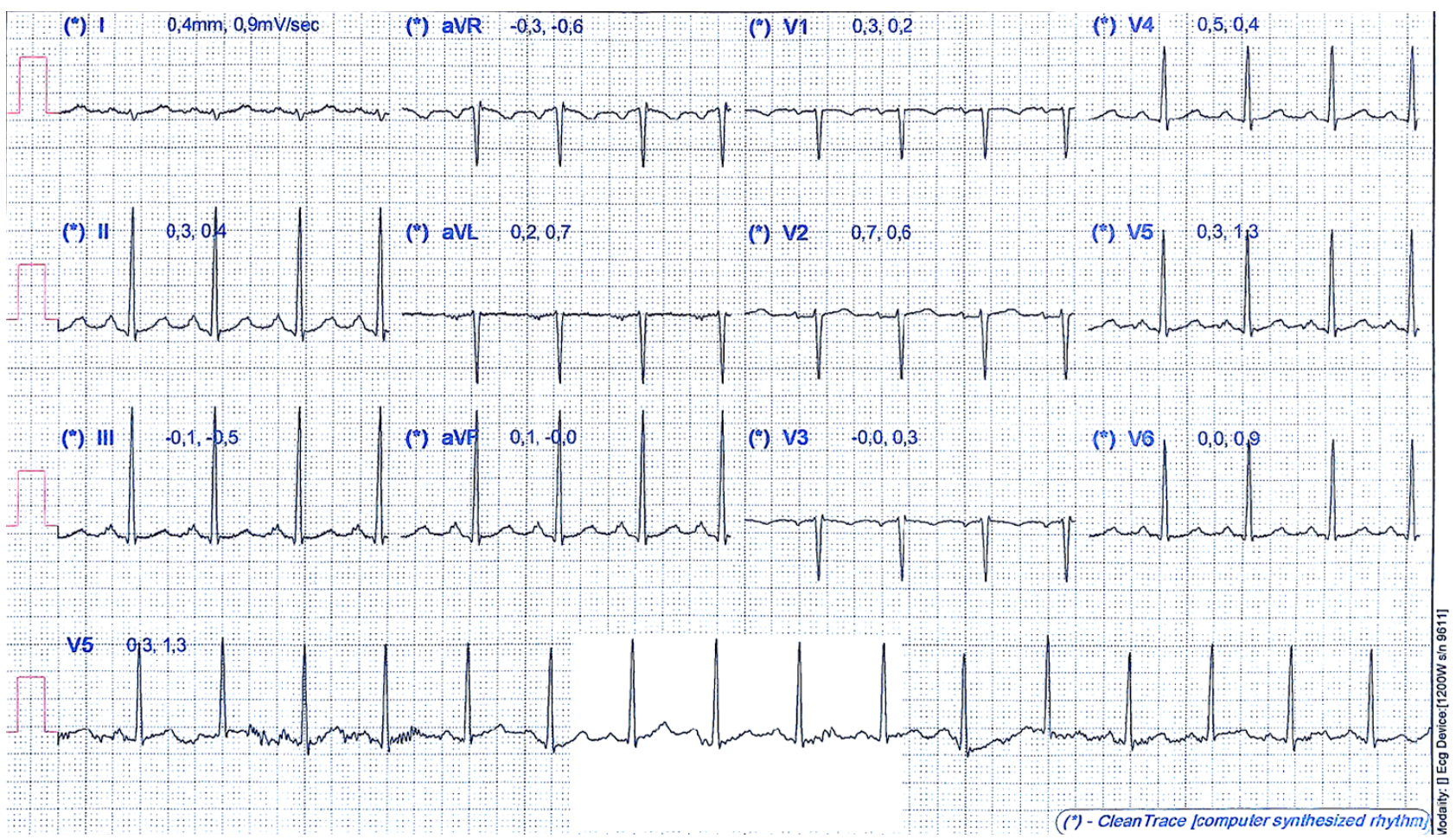

Figure 1 Baseline ECG recorded at rest before beginning exercise stress test.

catheter manipulation triggered incessant ectopic activity and monomorphic VT with identical morphology [12/12] to the previously observed during the exercise stress test. Anatomical reconstruction and voltage and activation maps were defined by using the CARTO-3 electroanatomic mapping system. No scars were detected. Precocity occurred in the posterior lateral wall of the RVOT, immediately below the pulmonary valve (Figure 3). Pace-mapping in this zone yielded a QRS morphology matching [12/12] to the spontaneous VT. Radiofrequency application in the arrhythmogenic focus with a discrete presystolic potential and a precocity of $34 \mathrm{~ms}$ immediately suppressed all ectopic activity despite maintaining isoproterenol infusion. Catheter ablation was completed with eight radiofrequency applications in adjacent areas. After 30 minutes, the effect was maintained, and the ectopic focus was successfully ablated, ending the procedure without complications.

The first follow-up examination obtained 1 month after the catheter ablation demonstrated normal resting 12-lead (Figure 4) and 24-h ECG. Exercise stress testing also elapsed normally [test results: heart rate $=173 \mathrm{bpm}$, power in watts $=171(2.9$ watts $/ \mathrm{kg}), 10 \mathrm{METS}$, and a $\mathrm{VO}_{2 \max }$ of $2.06 \mathrm{~L} / \mathrm{min}$ ] and the patient had no recurrent symptoms or episodes of $\mathrm{VT}$ at maximum efforts. The athlete was cleared for return to exercise, and at 3-month follow-up, the athlete was entirely asymptomatic with normal findings. All procedures performed in studies involving human participants were in accordance with the ethical standards of the institutional and/or national research committee(s) and with the Helsinki Declaration (as revised in 2013). Informed written consent was obtained from the patient to use her clinical data in this case report.

\section{Discussion}

We have presented a case of a 35-year-old highly-trained woman endurance athlete complaining about chest pain and dyspnea on exertion, dizziness and presyncope occurring during maximum-intensity exercise training sessions. She was misdiagnosed for 2 years until we finally diagnosed with RVOT-VT.

High-level endurance training has been associated with RV pathological remodeling, PVCs, and RVOT-VT (6). In pre-menopausal women, hormonal influences also seem to be significant and sympathetic adrenergic, peak systolic 


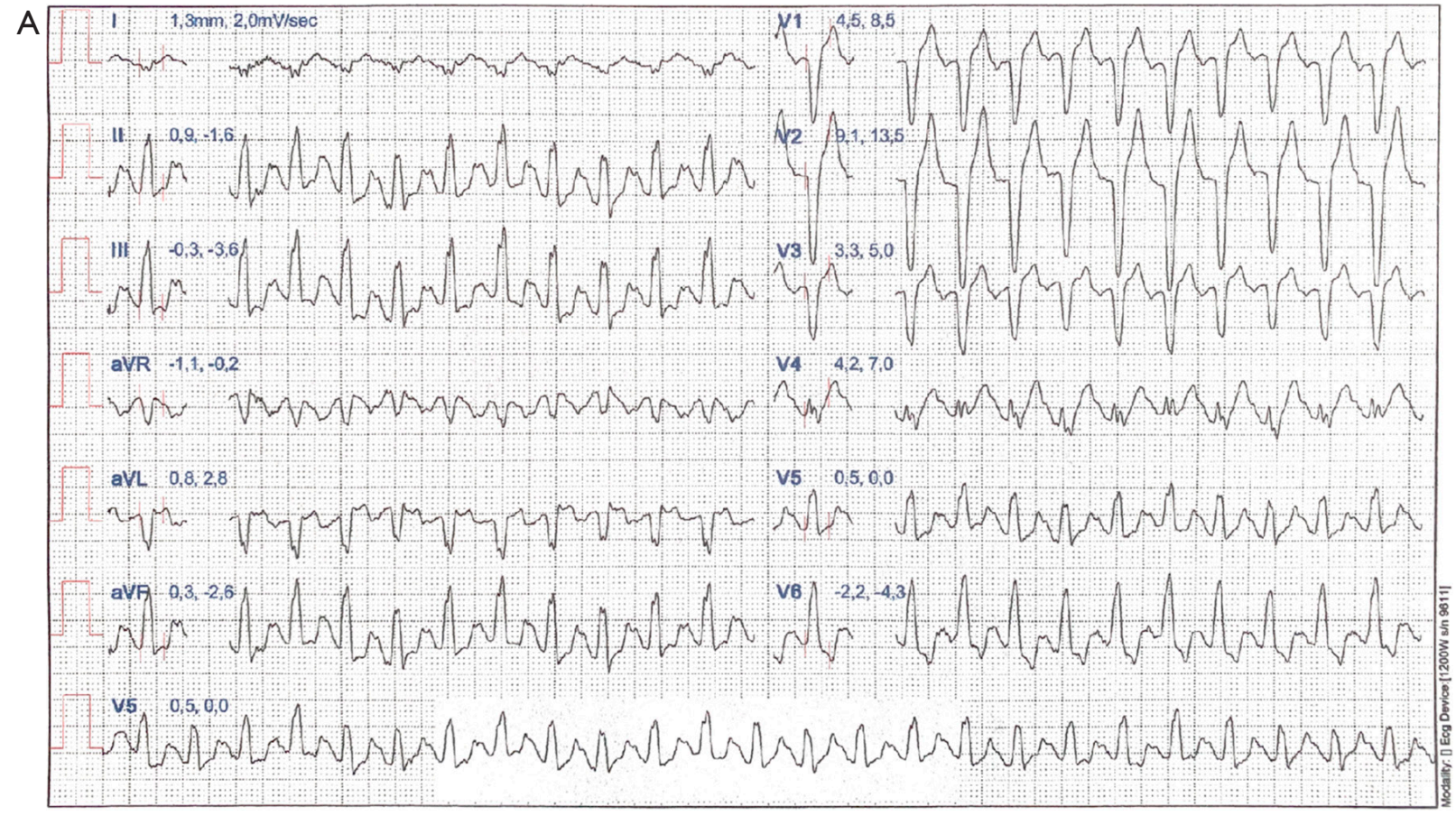

B

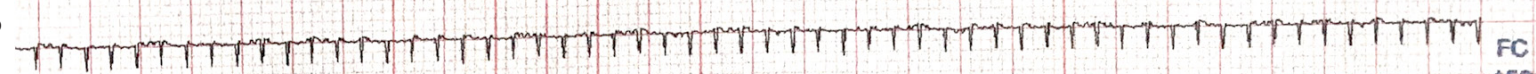

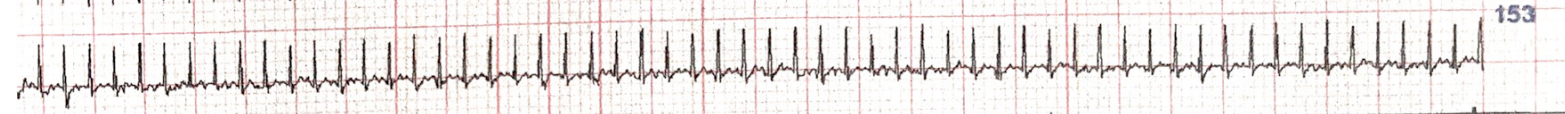

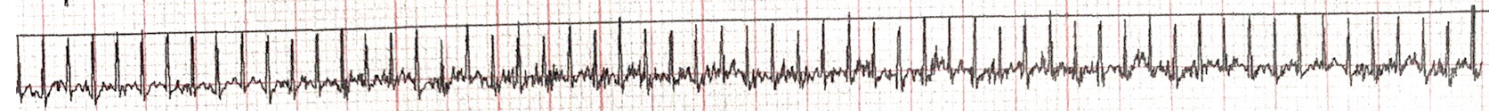

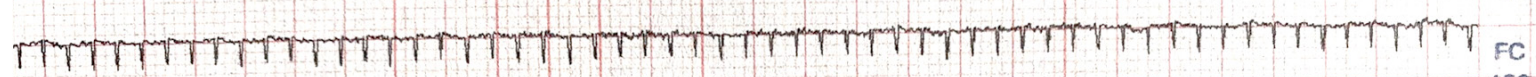

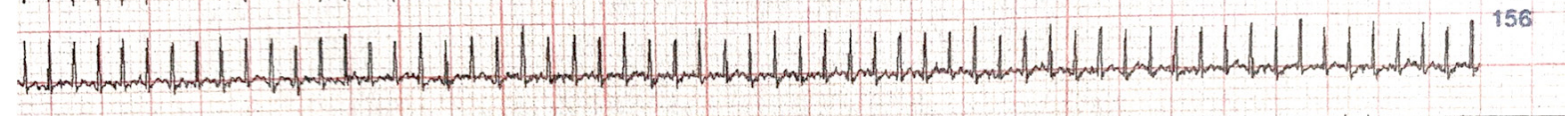

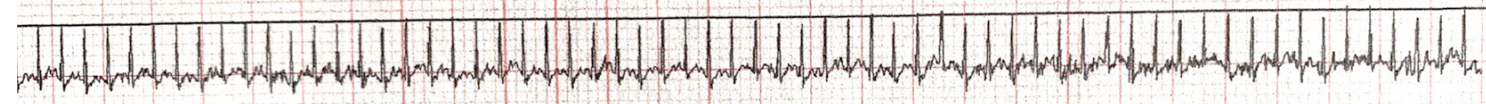
$2+08 \div 24$

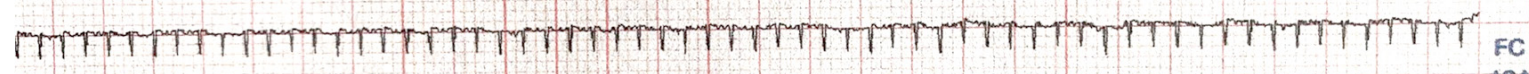

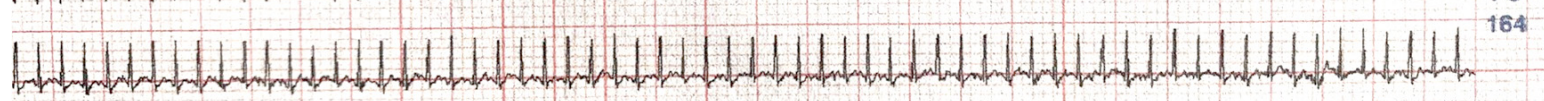

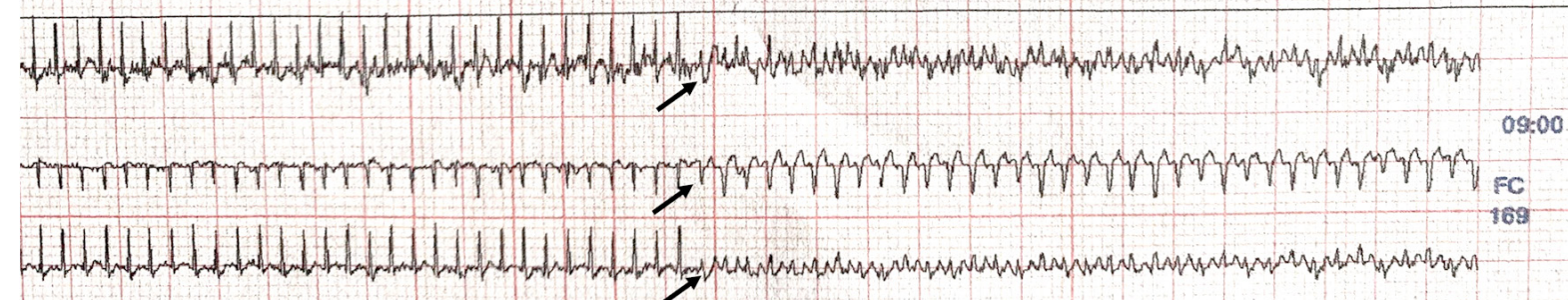

Figure 2 ECGs during the stress test. (A) ECG during the exercise stress test at maximal effort; (B) ECG demonstrating VT onset originating from the RVOT (169 bpm). The sustained ventricular tachycardia appeared spontaneously (arrows). ECG, electrocardiogram. 


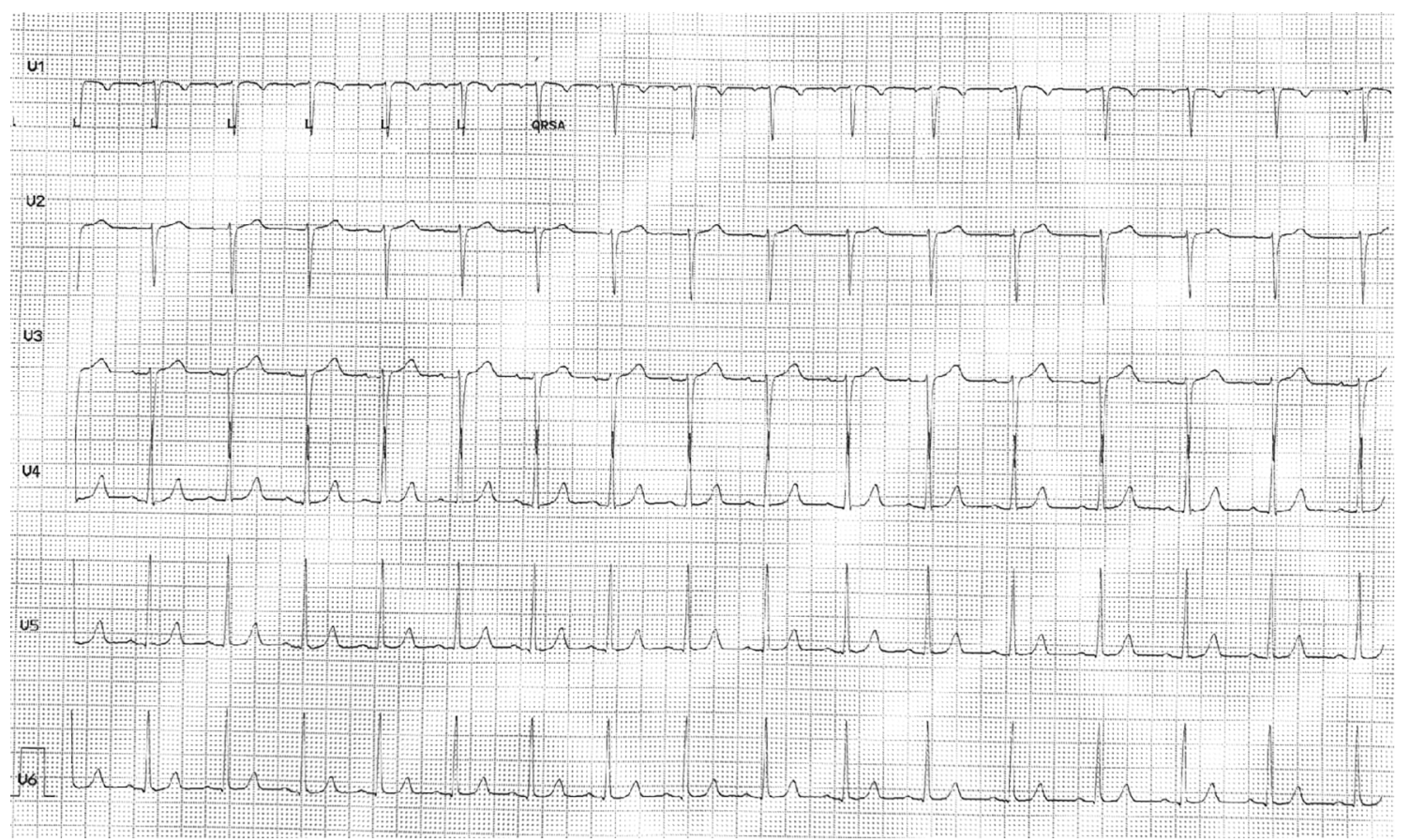

Figure 3 Baseline electrocardiogram (ECG) post-ablation.
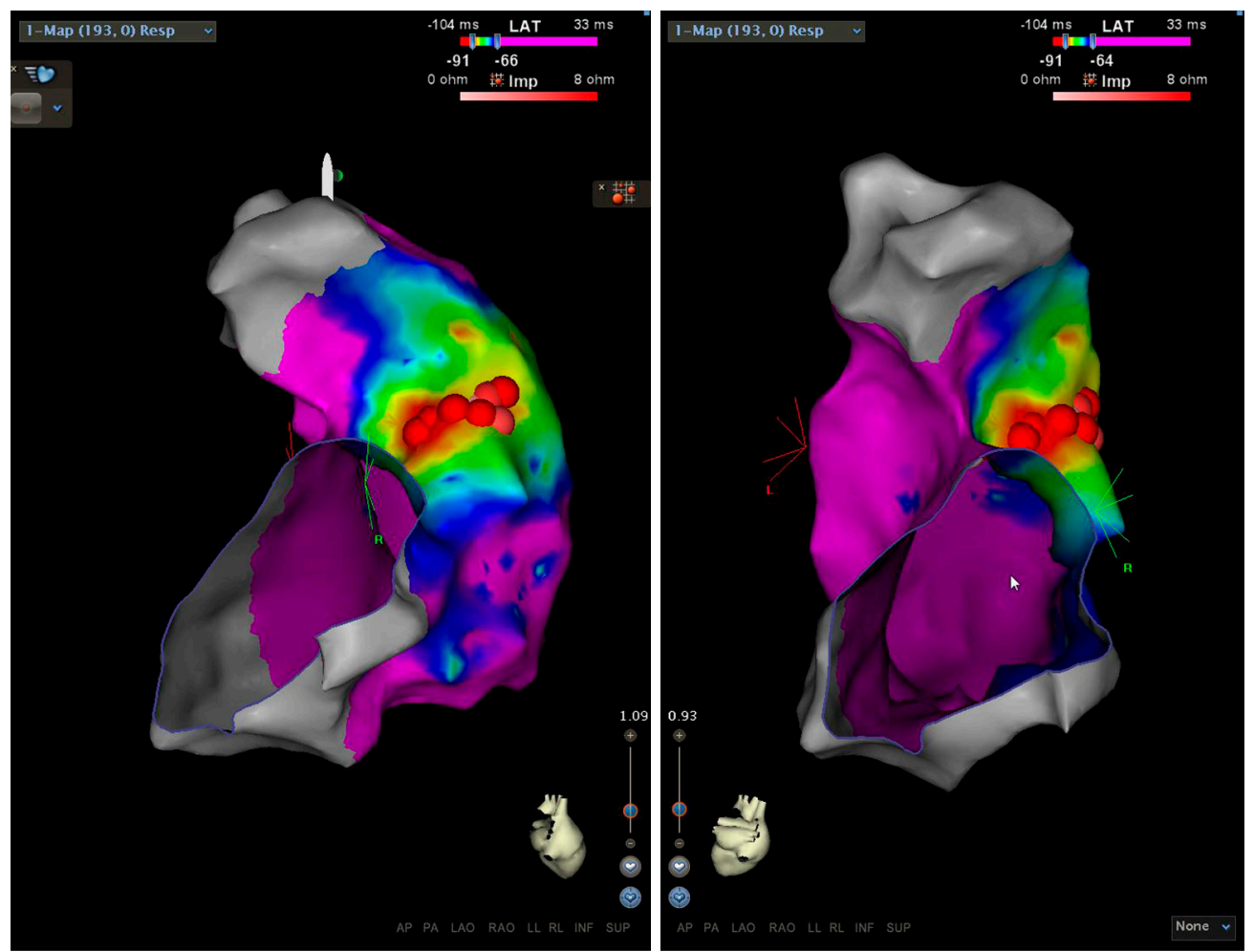

Figure 4 Right ventricular (RV) anatomic reconstruction and activation map during ventricular tachycardia (VT) acquired with the CARTO-3 system. The colors code shows the earliest zone in red (spreading after with rainbow degraded from red to violet) where the radiofrequency energy was administered (red points). 


\section{Page 6 of 8}

blood pressure, stroke volume, and peak VO2 modifications in response to exercise are slighter in female athletes (5). Then, cardiac structural and electrical changes resulting from long-term exercise training may be minimal, or even nonexistent, in women due to biological, anatomical, and hormonal characteristics (5). In effect, anterior $\mathrm{T}$ wave inversion is more common in females' athletes than in males, while voltage criteria for LV hypertrophy (LVH) and RV hypertrophy are less frequent in females (5). Female also tend to develop eccentric hypertrophy and RV enlargement is also more common in females (5). In brief, testosterone and dihydrotestosterone stimulate myocardial hypertrophy by directly acting on cardiac myocytes while estrogens reduce myocardial hypertrophy and cardiac fibroblasts proliferation (7). Androgenic and estrogenic receptors in myocytes modulate the expression of certain cardiac genes and sarcomeric proteins (8). Estrogens also increase nitric oxide concentrations, while estrogens and testosterone have contrary effects on angiotensin converting enzyme activity, which promote peripheral vasodilation and vasoconstriction with different effects on blood pressure (9). The activity of certain enzymes involved fatty acid oxidation and glucose uptake such as protein kinase B (AKT) and glycogen synthase kinase-3-beta (GSK3), is higher in females and might prevent the development of cardiac hypertrophy, which ultimately affect physiological cardiac hypertrophy adaptations (10). Besides, sex have an important role on the prevalence and expression of specific polymorphisms involved in the gene encoding for angiotensin and degree of LVH induced by exercise (11). This might explain, at least in part, sex differences in cardiac remodeling. In this regard, sex-differences in electroanatomical characteristics in patients with idiopathic RVOT-VT have been previously reported $(12,13)$.

The first evaluation recommended in suspected cases of RVOT-VT is the documentation of the ECG pattern of the VT performing a 12-lead ECG. In athletes with VT-associated symptoms with exertion, exercise stress testing has to be performed (14). Moreover, athletes having syncope, presyncope or palpitations with no arrhythmia detected on a single 12-lead ECG should undergo further evaluation with ambulatory ECG Holter monitoring (15). A small scar confined to the RVOT is unlikely to result in ECG abnormalities. Echocardiography is the first-line diagnostic imaging modality in patients with suspected normal heart VT and, in these cases, it focuses on confirming no subtle abnormalities of the heart, particularly the RV (16). In this case, no abnormalities were observed de la Guía-Galipienso et al. Ventricular tachycardia and exercise

neither in the baseline 12-lead ECG nor in ECG Holter monitoring nor in the echocardiogram. Only exercise stress testing induced the VT at maximum efforts.

A delayed-enhanced cMRI is also recommended in some cases in order to rule out arrhythmogenic cardiomyopathy. Besides, patients with a family history of sudden cardiac death and apparent RVOT tachycardia should be particularly evaluated by cardiac MRI. An isolated subepicardial RVOT scar should always be considered in high-level endurance athletes presenting with symptomatic VT (6). Endocardial bipolar voltage mapping has been recommended for detecting RVOT scars (17), particularly in women. Finally, patients who suffered an episode of unexplained sudden cardiac arrest secondary to a VT, coronary angiography can be used to confirm the presence or absence of ischemic heart disease (18). In our case, no active inflammation, fibrosis or scars were observed in LGE-MRI, and VT only emerged through electrophysiologic study and under isoproterenol infusion suggesting to depend of a delayed-activity mechanism.

The issue presented in this clinical case may be familiar to the electrophysiology community. However, this case report is relevant for physicians and other health professionals who are unfamiliar with sports medicine and sex differences in the cardiovascular system to understand the clinical implications of the present case. Although VT induced by long-term exercise is infrequent in females, we intended to underline that its incidence is changing. Importantly, sex differences in physiological cardiac adaptation to exercise are still unknown.

\section{Conclusions}

In this RVOT-VT case, results of baseline and ambulatory monitoring ECGs, as well as cardiac imaging techniques, were all negative. Exercise stress testing was crucial for the diagnosis, which was finally confirmed by endocardial unipolar voltage mapping. The recognition of this clinical entity in females may be challenging since cardiac remodeling in response to exercise may be invaluable due to their biological, anatomical, and hormonal characteristics. International recommendations are mostly based on male studies. However, electrical and structural adaptations in males and females may differ considerably. Women are increasingly participating in sports competitions and exercise training, and a better understanding of cardiac adaptations in response to exercise in females is mandatory. Finally, both exercise stress testing and diagnostic 
electrophysiological study represent essential and invaluable tools to reach a final diagnosis, especially in highly trained females. A delayed diagnosis has significant quality of life, diagnosis and therapeutic consequences, especially considering that limited endocardial ablation is potentially curative and with an excellent prognosis.

\section{Acknowledgments}

Funding: None.

\section{Footnote}

Reporting Checklist: The authors have completed CARE reporting checklist. Available at http://dx.doi.org/10.21037/ atm-20-3492

Conflicts of Interest: All authors have completed the ICMJE uniform disclosure form (available at: http://dx.doi. org/10.21037/atm-20-3492). FSG serves as Editorial Board Member for Annals of Translational Medicine with the terms of Appointment: Mar 2019-Feb 2021. The other authors have no conflicts of interest to declare.

Ethical Statement: The authors are accountable for all aspects of the work in ensuring that questions related to the accuracy or integrity of any part of the work are appropriately investigated and resolved. All procedures performed in studies involving human participants were in accordance with the ethical standards of the institutional and/or national research committee(s) and with the Helsinki Declaration (as revised in 2013). Written informed consent was obtained from the patient to use her clinical data in this case report.

Open Access Statement: This is an Open Access article distributed in accordance with the Creative Commons Attribution-NonCommercial-NoDerivs 4.0 International License (CC BY-NC-ND 4.0), which permits the noncommercial replication and distribution of the article with the strict proviso that no changes or edits are made and the original work is properly cited (including links to both the formal publication through the relevant DOI and the license). See: https://creativecommons.org/licenses/by-nc-nd/4.0/.

\section{References}

1. Fuenmayor AJ. Treatment Or Cure Of Right Ventricular
Outflow Tract Tachycardia. J Atr Fibrillation 2014;7:1038.

2. Pelliccia A, Sharma S, Gati S, et al. 2020 ESC Guidelines on sports cardiology and exercise in patients with cardiovascular disease. Eur Heart J 2021;42:17-96.

3. Finocchiaro G, Dhutia H, D'Silva A, et al. Effect of Sex and Sporting Discipline on LV Adaptation to Exercise. JACC Cardiovasc Imaging 2017;10:965-72.

4. D'Ascenzi F, Pisicchio C, Caselli S, et al. RV Remodeling in Olympic Athletes. JACC Cardiovasc Imaging 2017;10:385-93.

5. Colombo CSSS, Finocchiaro G. The Female Athlete's Heart: Facts and Fallacies. Curr Treat Options Cardiovasc Med 2018;20:101.

6. Venlet J, Piers SR, Jongbloed JD, et al. Isolated Subepicardial Right Ventricular Outflow Tract Scar in Athletes With Ventricular Tachycardia. J Am Coll Cardiol 2017;69:497-507.

7. Marsh JD, Lehmann MH, Ritchie RH, et al. Androgen receptors mediate hypertrophy in cardiac myocytes. Circulation 1998;98:256-61.

8. Grohé C, Kahlert S, Löbbert K, et al. Cardiac myocytes and fibroblasts contain functional estrogen receptors. FEBS Lett 1997;416:107-12.

9. Vega RB, Konhilas JP, Kelly DP, et al. Molecular Mechanisms Underlying Cardiac Adaptation to Exercise. Cell Metab 2017;25:1012-26.

10. Foryst-Ludwig A, Kreissl MC, Sprang C, et al. Sex differences in physiological cardiac hypertrophy are associated with exercise-mediated changes in energy substrate availability. Am J Physiol Heart Circ Physiol 2011;301:H115-22.

11. Diet F, Graf C, Mahnke N, et al. ACE and angiotensinogen gene genotypes and left ventricular mass in athletes. Eur J Clin Invest 2001;31:836-42.

12. Liao JN, Hu YF, Lin WS, et al. Gender Difference in Idiopathic Right Ventricular Outflow Tract-Ventricular Tachycardia. Acta Cardiol Sin 2013;29:304-10.

13. Yang SG, Mlček M, Kittnar O. Gender differences in electrophysiological characteristics of idiopathic ventricular tachycardia originating from right ventricular outflow tract. Physiol Res 2014;63:S451-8.

14. Elhendy A, Chandrasekaran K, Gersh BJ, et al. Functional and prognostic significance of exercise-induced ventricular arrhythmias in patients with suspected coronary artery disease. Am J Cardiol 2002;90:95-100.

15. Barrett PM, Komatireddy R, Haaser S, et al. Comparison of 24-hour Holter monitoring with 14-day novel adhesive patch electrocardiographic monitoring. Am J Med 
Page 8 of 8

2014;127:95.e11-7.

16. Whyte GP, Stephens N, Senior R, et al. Differentiation of RVOT-VT and ARVC in an elite athlete. Med Sci Sports Exerc 2008;40:1357-61.

17. Marra MP, Leoni L, Bauce B, et al. Imaging study of ventricular scar in arrhythmogenic right ventricular cardiomyopathy: comparison of 3D standard electroanatomical voltage mapping and contrast-enhanced cardiac magnetic resonance. Circ Arrhythm Electrophysiol 2012;5:91-100.

Cite this article as: de la Guía-Galipienso F, Sanchis-Gomar F, Quesada-Dorador A. Diagnostic electrophysiological study in a highly trained young woman with presyncopal symptoms during exercise: a case report. Ann Transl Med 2021;9(2):177. doi: 10.21037/atm-20-3492 de la Guía-Galipienso et al. Ventricular tachycardia and exercise

18. Al-Khatib SM, Stevenson WG, Ackerman MJ, et al. 2017 AHA/ACC/HRS Guideline for Management of Patients With Ventricular Arrhythmias and the Prevention of Sudden Cardiac Death: Executive Summary: A Report of the American College of Cardiology/American Heart Association Task Force on Clinical Practice Guidelines and the Heart Rhythm Society. Circulation 2018;138:e210-71. Erratum in: Circulation. 2018 Sep 25;138(13):e415-e418. doi: 10.1161/CIR.0000000000000615. 